\title{
Study on the correlation between the levels of homocysteine, blood lipids and inflammatory markers and the carotid plaque in patients with ischemic cerebrovascular disease.
}

\author{
Weibo Zheng*, Wenfang Xu, Xiaohua Wang, Jiangfeng Chen, Qiong Tan \\ Department of Clinical Laboratory, Shaoxing Municipal Hospital, Zhejiang, PR China
}

\begin{abstract}
Objective: To investigate the correlation between the levels of homocysteine, blood lipids and inflammatory markers and the carotid plaque in patients with ischemic cerebrovascular disease.

Methods: 56 cases of patients with ischemic cerebrovascular disease admitted by this hospital from October 2010 to October 2013 were categorized as a case group while 56 cases of healthy physical examinees of similar age and sex ratio were chosen as the control group. Based on color Doppler ultrasound results in carotid artery, the two groups were subdivided into carotid plaque group and noplaque group respectively. Levels of homocysteine, blood lipids and inflammatory markers of every group were measured.

Results: The case number of carotid plaque and IMT (Intimal Medial Thickness) in the case group is larger than those of the control group, which is statistically significant in difference $(P<0.05)$. Levels of blood lipid TC, LDL and three inflammatory markers (Hs-CRP, IL-6 and Fbg) and homocysteine in the case group are higher than those in control group, which is statistically significant in differences $(P<0.05)$. Levels of Lipids TC, LDL and three inflammatory markers (Hs-CRP, IL-6 and Fbg) and homocysteine in the plaque group of the case group are higher than those in the no-plaque group of the case group, which is statistically significant in differences $(P<0.05)$. Levels of Lipids TC, LDL and three inflammatory markers (Hs-CRP, IL-6 and Fbg) and homocysteine in the plaque group of control group are higher than those in the no-plaque group of control group, which is statistically significant in differences $(\mathbf{P}<0.05)$. Levels of Lipids TC, LDL and three inflammatory markers (Hs-CRP, IL-6 and Fbg) and homocysteine of the plaque group are higher than those in the no-plaque group of the case group, which is statistically significant in differences $(P<0.05)$.

Conclusion: Levels of homocysteine, blood lipids, and inflammatory markers in patients with ischemic cerebrovascular disease are closely associated with different properties of carotid plaque.
\end{abstract}

Keywords: Homocysteine, Hs-CRP, IL-6, Fbg.

Accepted on March 9, 2017

\section{Introduction}

Ischemic Cerebrovascular Disease (ICVD) is a common disease in the nervous system leading to hospitalization. Generally, ICD does not require an intensive care unit unless extenuating circumstances are faced. The main related symptoms are impaired consciousness with or without cerebral edema, crescendo transient ischemic attacks or evolving stroke. The treatment of ICD is categorized as preventive, supportive, standard interventions and newer experimental approaches. Patients with it are prone to relapse and have a rather high rate of mortality and morbidity [1,2]. With the improvement of people's living standard, unhealthy habits in life and diet lead to a general decline in physical fitness, increasing the incidence of cerebrovascular disease in China year by year. According to statistics, cerebrovascular disease is the number one killer in China, which poses a serious threat to the health of the Chinese people and brings huge financial burden to the families [3]. Ischemic cerebrovascular disease are the majority, of which about $1 / 3$ are caused by carotid plaque lesions [4]. For a long time, there are two theories about the mechanism for ischemic cerebrovascular disease induced by carotid artery lesions, namely, theories on low perfusion in downstream tissues of hemodynamics and thromboembolism [5]. In the past, based on the understanding of theory on low perfusion in downstream tissues of hemodynamics, doctors in general focus only on the degree of carotid artery stenosis and its treatment in patients with ischemic cerebrovascular disease along with carotid artery lesions [6]. Presently, with the advancement in the study, it's believed that the main reason for cerebrovascular disease is the embolus due to the rupture of carotid plaque [6]. Therefore, an early detection and evaluation on characteristics of carotid plaque and degree of carotid artery stenosis, and early discovery of vulnerable plaque, are extremely important for the 
prevention on occurrence of ischemic cerebrovascular disease [7]. Related literatures abroad have confirmed that ischemic cerebrovascular disease in Europe and the United States are closely related to carotid plaques, although whether the case is the same in China is still in comparatively significant controversy. It is worth mentioning that in many domestic literatures, patients with cerebrovascular disease suffer higher abnormal rate of carotid arteries and detection rate of sclerotic plaque $[8,9]$. Clinical studies indicates that carotid plaques in patients are predominantly a single large one with instability and other characteristics, which leads to rupture of the plaque in the neck or varying degrees of vascular stenosis, resulting in ischemic cerebrovascular disease [10]. Some patients are with multiple small calcified plaques characterized by stability, rarely leading to clinical symptoms. Relevant research shows that carotid plaque formation and carotid intimal thickening are closely related to ischemic cerebrovascular disease. Moreover, some risk factors are closely associated with atherosclerosis. Timely elimination or reduction of such factors can slow down the progression of atherosclerosis, thereby changing the current situation of cerebral infarction treatment [11]. Currently, studies have shown that plasma homocysteine levels are closely connected to the occurrence of pulmonary embolism, ischemic cerebrovascular disease and peripheral vascular disease. However, its mechanism of action in the process of ischemic cerebrovascular disease and atherosclerosis, and whether it's associated with cerebrovascular disease induced by unstable plaques, are not yet fully clear [12]. Abnormal blood lipid is considered to be an important cause of the formation of atherosclerosis. Whereas, the current research shows that in addition to total cholesterol, as one of the indicators of blood lipid level, which is comparatively consistent with the relevance of atherosclerosis, other indicators are not uniform [13]. In the whole process of atherosclerotic plaque formation, inflammatory mediators are involved. Inflammation plays an important role in the development of atherosclerosis. To explore the factors related to the pathogenesis of cerebrovascular disease for the improvement of the theoretical basis in preventing and controlling cerebrovascular diseases, this study selected 56 cases of patients with ischemic cerebrovascular disease and 56 cases of healthy physical examinees as the research objects who are divided into different groups to investigate the correlation between the levels of homocysteine, lipid and inflammatory markers and the carotid plaque formation in patients with ischemic cerebrovascular disease.

\section{Material and Methods}

\section{Research material}

56 cases of patients with ischemic cerebrovascular disease admitted by this hospital from October 2010 to October 2013 were selected as the research objects (30 males and 26 females), aged from 37 to 84 years and a mean age of $56.4 \pm$ 10.1 years. Diagnostic criteria for ischemic encephalopathy refer to the standards formulated by the Fourth National Conference on Cerebrovascular Disease. Patients were confirmed by CT or MRI examination on the head and those who are suffered from stroke, brain hemorrhage and hemorrhagic infarction were excluded. Patients with cerebral infarction were 29 cases, vertebrobasilar insufficiency 22 cases and transient ischemic onset 5 cases. Another 56 cases of healthy physical examinees of similar age and sex ratio as the case group were categorized as the control group. Sex ratio, age, disease classification and other information between the case group and the control group displayed no significant difference, which was comparable then. Based on color Doppler ultrasound results, the case group was subdivided into the plaque group and the no-plaque group, and the control group was separated into plaque group and no- plaque group, too. Besides, plaque groups of both the case group and the control group collectively made up the plaque group, while the no-plaque groups of both groups were collectively called the no-plaque group.

\section{Methods}

Carotid ultrasonography: Philips HDI-5000 Color ultrasound device with $7 \mathrm{MHz}$ double deep pulse probe was use for carotid ultrasonography within $24 \sim 72 \mathrm{~h}$ following patients' admission to hospital. Detection methods: the patients lied on their back with the head backward and turning to the opposite side of the inspection area. The front part of the neck (close to throat) was exposed fully and longitudinal scan from the base of the neck to the long axis of the carotid artery was executed. The main checkpoints were the beginning part of the Internal Carotid Artery (ICA), the carotid artery Bifurcation (BIF) and distal end of Common Carotid Artery (CCA). When IMT (intimal medial thickness) $\leq 1.0 \mathrm{~mm}$, it is normal; IMT (Intimal Medial Thickness $)=1.0-1.2 \mathrm{~mm}$, carotid intimal thickening; IMT(intimal medial thickness) $\geq 1.2 \mathrm{~mm}$, carotid plaque formation.

Determination of blood lipid components: Patients' venous blood was collected after $12 \mathrm{~h}$ of fasting and blood lipid components through application of automatic biochemical analyzer were determined, with the Total Cholesterol (TC), High Density Lipoprotein Cholesterol (HDL-C), Low-Density Lipoprotein Cholesterol (LDL-C) and Triglyceride (TG) included.

Determination of Inflammatory markers and homocysteine concentrations: $6 \mathrm{ml}$ of patients' fasting blood was collected. The measurement of serum markers Hs-CRP was implemented by means of immune turbidimetry, with the range of reference value at $0 \sim 0.8 \mathrm{mg} / \mathrm{L}$; IL- 6 by ELISA at $24 \sim 58 \mathrm{pg} / \mathrm{ml}$; detection of Fbg by thrombin ranging between $1.80 \sim 3.80 \mathrm{~g} / \mathrm{L}$; homocysteine by quantitative fluorescence method from 5.0 to $15.0 \mu \mathrm{mol} / \mathrm{L}$.

\section{Results}

SPSS19.0 software for data analysis, chi-square test for the comparison of enumeration data and t-test for the comparison of measurement data $(\alpha=0.05)$ were adopted. When $\mathrm{P}<0.05$, the difference was statistically significant. 
Study on the correlation between the levels of homocysteine, blood lipids and inflammatory markers and the carotid plaque in patients with ischemic cerebrovascular disease

\section{Comparison of case number of carotid plaque and IMT between the case group and the control group}

The case number of carotid plaque and IMT in the case group are larger than those in the control group, which is statistically significant in differences $(\mathrm{P}<0.05)$ (Table 1).

Table 1. Comparison of case number of carotid plaque and IMT between case group and control group.

\begin{tabular}{llllll}
\hline Groups & Case number $(\mathbf{n})$ & $\begin{array}{l}\text { Carotid } \\
(\mathbf{n})\end{array}$ & artery & plaque & IMT $(\mathbf{m m})$ \\
\hline case group & 56 & $44^{*}$ & 1.39 & \pm \\
& & & $0.14^{*}$ & \\
\hline Control Group & 56 & 11 & $1.22 \pm 0.10$
\end{tabular}

Note: *denotes that $\mathrm{P}<0.05$ compared with control group.

Comparison of the levels of homocysteine, blood lipids and inflammatory markers between the case group and the control group

Levels of blood lipid TC, LDL and three inflammatory markers (Hs-CRP, IL-6, Fbg) and homocysteine in case group are higher than those in control group, which is statistically significant in differences $(\mathrm{P}<0.05)$ (Table 2$)$.

Table 2. Comparison of the levels of homocysteine, blood lipids and inflammatory markers between the case group and the control group.

\begin{tabular}{lll}
\hline Index & Case group $(\mathbf{n = 5 6})$ & Control group $(\mathbf{n = 5 6})$ \\
\hline TC $(\mathrm{mmol} / \mathrm{L})$ & $5.36 \pm 1.04^{*}$ & $4.49 \pm 0.91$ \\
\hline $\mathrm{TG}(\mathrm{mmol} / \mathrm{L})$ & $1.63 \pm 0.89$ & $1.54 \pm 0.71$ \\
\hline $\mathrm{HDL}(\mathrm{mmol} / \mathrm{L})$ & $1.44 \pm 0.55$ & $1.50 \pm 0.62$ \\
\hline $\mathrm{LDL}(\mathrm{mmol} / \mathrm{L})$ & $3.32 \pm 0.85^{*}$ & $2.78 \pm 0.88$ \\
\hline $\mathrm{Hs}-\mathrm{CRP}(\mathrm{mg} / \mathrm{L})$ & $5.18 \pm 2.22^{*}$ & $2.29 \pm 0.98$ \\
\hline $\mathrm{LL}-6(\mathrm{pg} / \mathrm{L})$ & $74.87 \pm 28.98^{*}$ & $55.48 \pm 19.68$ \\
\hline Fbg $(\mathrm{g} / \mathrm{L})$ & $3.39 \pm 0.99^{*}$ & $2.55 \pm 0.89$ \\
\hline $\mathrm{HCY}(\mu \mathrm{mol} / \mathrm{L})$ & $23.31 \pm 2.96^{*}$ & $12.89 \pm 4.21$ \\
\hline Note: ${ }^{*}$ denotes that $\mathrm{P}<0.05$ compared with control group.
\end{tabular}

\section{Comparison of the levels of homocysteine, lipid and inflammatory markers between every subgroup of both the case group and the control group}

Levels of Lipids TC, LDL and three inflammatory markers (Hs-CRP, IL-6 and Fbg) and homocysteine in plaque group of case group are higher than those in the no-plaque group of the case group, which was statistically significant in the differences $(\mathrm{P}<0.05)$. Levels of Lipids TC, LDL and three inflammatory markers (Hs-CRP, IL-6, Fbg) and homocysteine in the plaque group of the control group are higher than those in the no-plaque group of the control group, which is statistically significant in differences $(\mathrm{P}<0.05)$ (Table 3$)$.

Table 3. Comparison of the levels of homocysteine, lipid and inflammatory markers between every subgroup of both the case group and the control group.

\begin{tabular}{|c|c|c|c|c|}
\hline \multirow[t]{2}{*}{ Index } & \multicolumn{2}{|l|}{ Case group $(n=56)$} & \multicolumn{2}{|l|}{ Control group $(n=56)$} \\
\hline & Plaque group $(n=44)$ & No plaque group $(n=12)$ & Plaque group $(n=11)$ & No plaque group $(n=45)$ \\
\hline $\mathrm{TC}(\mathrm{mmol} / \mathrm{L})$ & $4.96 \pm 1.01^{*}$ & $4.65 \pm 0.88$ & $4.62 \pm 0.85^{\star *}$ & $4.08 \pm 0.78$ \\
\hline TG (mmol/L) & $1.65 \pm 0.86$ & $1.53 \pm 0.73$ & $1.57 \pm 0.61$ & $1.50 \pm 0.59$ \\
\hline $\mathrm{HDL}(\mathrm{mmol} / \mathrm{L})$ & $1.42 \pm 0.53$ & $1.48 \pm 0.60$ & $1.49 \pm 0.63$ & $1.51 \pm 0.66$ \\
\hline $\mathrm{LDL}(\mathrm{mmol} / \mathrm{L})$ & $3.40 \pm 0.86^{*}$ & $2.95 \pm 0.84$ & $2.94 \pm 0.91^{* *}$ & $2.69 \pm 0.85$ \\
\hline Hs-CRP (mg/L) & $5.86 \pm 2.36^{*}$ & $4.29 \pm 0.88$ & $2.69 \pm 0.96^{* *}$ & $2.25 \pm 0.78$ \\
\hline IL-6 (pg/L) & $72.84 \pm 26.91^{*}$ & $65.58 \pm 20.34$ & $57.89 \pm 19.88^{\star *}$ & $53.24 \pm 18.67$ \\
\hline $\mathrm{Fbg}(\mathrm{g} / \mathrm{L})$ & $3.49 \pm 1.02^{*}$ & $2.94 \pm 0.91$ & $2.85 \pm 0.94^{* *}$ & $2.34 \pm 0.78$ \\
\hline $\mathrm{HCY}(\mu \mathrm{mol} / \mathrm{L})$ & $23.85 \pm 2.88^{*}$ & $20.85 \pm 2.58$ & $18.26 \pm 4.35^{* *}$ & $12.11 \pm 3.68$ \\
\hline
\end{tabular}

Note: "denotes that $\mathrm{P}<0.05$ compared with no plaque group of case group; ${ }^{*}$ indicates that $\mathrm{P}<0.05$ compared with no plaque group of control group.

Comparison of levels of homocysteine, lipid and inflammatory markers between the plaque group and the no-plaque group

Levels of Lipids TC, LDL and three inflammatory markers (Hs-CRP, IL-6 and Fbg) and homocysteine in the plaque group are higher than those of the no-plaque group, which is statistically significant in differences $(\mathrm{P}<0.05)$ (Table 4$)$.

Table 4. Comparison of levels of homocysteine, lipid and inflammatory markers between the plaque group and the no-plaque group.

\begin{tabular}{lll}
\hline Index & Plaque group $(\mathbf{n}=55)$ & No plaque group $(\mathbf{n}=57)$ \\
\hline TC $(\mathrm{mmol} / \mathrm{L})$ & $5.26 \pm 1.24^{*}$ & $4.48 \pm 0.89$ \\
\hline
\end{tabular}




\begin{tabular}{lll}
\hline TG $(\mathrm{mmol} / \mathrm{L})$ & $1.74 \pm 0.91$ & $1.52 \pm 0.81$ \\
\hline $\mathrm{HDL}(\mathrm{mmol} / \mathrm{L})$ & $1.41 \pm 0.52$ & $1.48 \pm 0.66$ \\
\hline $\mathrm{LDL}(\mathrm{mmol} / \mathrm{L})$ & $3.42 \pm 0.65^{*}$ & $2.81 \pm 0.90$ \\
\hline $\mathrm{Hs}-\mathrm{CRP}(\mathrm{mg} / \mathrm{L})$ & $5.69 \pm 2.32^{*}$ & $2.86 \pm 1.08$ \\
\hline $\mathrm{IL}-6(\mathrm{pg} / \mathrm{L})$ & $71.85 \pm 25.94^{*}$ & $60.45 \pm 17.65$ \\
\hline $\mathrm{Fbg}(\mathrm{g} / \mathrm{L})$ & $3.51 \pm 1.02^{*}$ & $2.84 \pm 0.88$ \\
\hline $\mathrm{HCY}(\mu \mathrm{mol} / \mathrm{L})$ & $23.96 \pm 3.12^{*}$ & $14.65 \pm 4.88$ \\
\hline
\end{tabular}

Note: "denotes that $\mathrm{P}<0.05$ compared with the control group.

\section{Discussion}

Causes for carotid atherosclerosis are considerable. It can reflect the situation of systemic atherosclerosis. One of the main causes of ischemic cerebrovascular disease is carotid atherosclerotic plaque whose characteristics tightly bond with the occurrence of ischemic cerebrovascular disease [14]. Atherosclerosis can cause vascular wall lesions, resulting in stenosis, thrombosis or closure of the lumen. Afterwards, localized blockage of blood supply in brain tissue occurs, which leads to its necrosis as a result of ischemia and hypoxia. Eventually, neurological symptoms arise. The pathological feature of atherosclerosis is the deposition of the lipid from the blood in the arterial intima, resulting in the focal fibrous thickening of the intima and tissue necrosis and disintegration deep in the intima, thus forming plaques. These plaques impede arteries over time, affecting blood supply [15]. Cerebral artery and carotid artery are integral components of systemic vascular bed, both of which share sameness in pathogenesis and risk factors of atherosclerosis. Generally, pathological change process of atherosclerosis develops with three stages, namely, beginning with vascular endothelial dysfunction, developing thickened vascular intima, forming atherosclerotic plaques [16]. The occurrence of carotid plaques is associated with many factors. Research on the risk factors for carotid plaque formation is clinically important for the prevention and control of the ischemic cerebrovascular disease. Clinical study reveals that carotid intimal thickening, atheromatous plaque formation and arterial stenosis are closely associated with ischemic cerebrovascular disease. And diabetes, hypertension, hyperlipidemia, obesity, alcoholism, smoking and so forth are common risk factors of atherosclerosis [17].

In recent years, studies indicate that abnormal blood lipid components may be an important reason of atherosclerosis [18]. Dyslipidemia causes endothelial cell damage, which increases vascular wall permeability so that plasma proteins can enter into the intima. That stimulates the elimination response of macrophages as well as proliferation of vascular smooth muscle cells, in the end, leading to plaque formation [19]. In the study on correlation between atherosclerosis and blood lipid components, Total Cholesterol (TC) is comparatively consistent with the development of atherosclerosis [20]. TC (Total Cholesterol) is the main component of CAS plaque. Excessive TC in plasma in the form of LDL (Low Density Lipoprotein) combines with mucopolysaccharide on the arterial wall to produce insoluble precipitate, resulting in proliferation of the fiber group and ending up with the formation of CAS plaque [21]. Besides, oxidized LDL (Low Density Lipoprotein) can cause endothelial cell damage, promoting adhesion between monocyte and endothelial cells, which accelerates the formation of CAS plaque. In this study, the comparison between the case group and the control group along with between the plaque group and the no-plaque group showed that levels in TC and LDL in the case group and the plaque group were significantly increased. The difference was statistically significant $(\mathrm{P}<0.05)$. Levels in $\mathrm{TC}$ and $\mathrm{LDL}$ in the plaque group were higher than those in the no-plaque group $(\mathrm{P}<0.05)$, which reveals the close association between the elevation of $\mathrm{TC}$ and LDL in the plasma and carotid plaque formation.

In addition to abnormal blood lipid components, more and more research has begun to focus on the role of inflammatory mediators in ischemic cerebrovascular diseases. In the course of atherosclerosis, inflammation plays an important role since inflammatory mediators involve in the formation, development and rupture of plaque [22]. Hs-CRP is an acute phase reactant which is recognized as inflammatory markers closely linking with atherosclerosis. IL-6 mainly generated by activated macrophages or monocytes can prompt platelet aggregation, and cause proliferation of vascular smooth muscle cell, which accelerates the progression of atherosclerosis, or cause the unstable development of plaques. Fbg promotes blood clotting, fiber plaque deposition, platelet aggregation and vascular smooth muscle cell proliferation. Furthermore, Fbg increases vascular permeability, causing fibroblast proliferation and migration to intima and stimulating plaque formation. The results of this study shows that levels of three kinds of inflammatory markers (Hs-CRP, IL-6 and Fbg) in case group are higher than those in control group, and the difference was statistically significant $(\mathrm{P}<0.05)$. Levels of three kinds of inflammatory markers (Hs-CRP, IL-6 and Fbg) in plaque groups of both the case group and the control group are higher than those in the no-plaque group $(\mathrm{P}<0.05)$, which prompts the correlation between three kinds of inflammatory markers (HsCRP, IL-6 and Fbg) and the formation of carotid plaque in ischemic cerebrovascular disease.

\section{Conclusion}

Homocysteine is a metabolite of methionine. Numerous studies in recent years have shown that it is closely related to and an important risk factor of atherosclerosis [23]. This study indicates that homocysteine level in case group are higher than those in the control group, which is statistically significant in difference $(\mathrm{P}<0.05)$. Homocysteine level in plaque groups of both the case group and the control group are higher than those in the no-plaque group $(\mathrm{P}<0.05)$, which prompts the correlation between homocysteine and the formation of carotid artery plaque in ischemic cerebrovascular disease, forming an important risk factor of carotid artery plaque in ischemic cerebrovascular disease. This is probably because 
homocysteine causes endothelial cell injury and dysfunction, which stimulates proliferation of vascular smooth muscle cell, destroys the balance of coagulation and fibrinolysis system and imposes impact on lipid metabolism, therefore the body is in the prothrombotic phase causing atherosclerosis and ending up with ischemic cerebrovascular disease.

\section{References}

1. Luo Y, Li J, Guan D. Role of abnormal lipid component in carotid plaque formation for patients with ischemic cerebrovascular disease. Chinese J Gerontol 2009; 29: 3280-3281.

2. Hira RS, Pokharel Y, Cowart J, Akeroyd JM, Ramsey D, Nambi V, Petersen LA. Compliance with risk factor optimization and medical therapy in patients with peripheral vascular disease (peripheral artery and ischemic cerebrovascular disease) compared to ischemic heart disease. Circulation Cardiovascular Quality Outcomes 2015; 8: 11.

3. Luo GG, Han JF, Lin W. Relationship between plasma homocysteine and carotid atherosclerotic plaques in patients with ischemic cerebrovascular disease. Chinese J Cerebrovasc Dis 2012; 9: 123-127.

4. Kohsaka S, Jin Z, Rundek T, Homma S, Sacco RL, Di Tullio MR. Relationship between serum lipid values and atherosclerotic burden in the proximal thoracic aorta. Int J Stroke 2010; 5: 257-263.

5. Alvarez B, Yugueros X, Fernandez E, Luccini F, Gene A, Matas M. Relationship between plasma homocysteine and the morphological and immunohistochemical study of carotid plaques in patients with carotid stenosis over $70 \%$. Ann Vasc Surg 2012; 26: 500-505.

6. Lopez OL, Becker JT, Kuller LH. Patterns of compensation and vulnerability in normal subjects at risk of Alzheimers disease. J Alzheimers Dis 2013; 33: 427-438.

7. Cruz DN. Clinical Chemistry and Laboratory Medicine (CCLM). Clin Chem Lab Med 2012; 50: 1533-1545.

8. Ohkuma T, Minagawa T, Takada N, Ohno M, Oda H, Ohashi H. C-reactive protein, lipoprotein (a), homocysteine, and male sex contribute to carotid atherosclerosis in peritoneal dialysis patients. Am J Kidney Dis 2003; 42: 355-361.

9. Xu WH, Dong C, Rundek T, Elkind MS, Sacco RL. Serum albumin levels are associated with cardioembolic and cryptogenic ischemic strokes Northern Manhattan study. Stroke 2014; 45: 973-978.

10. Gu X, Shen L, Zhou Y. Correlation between levels of homocysteine, blood lipids and inflammatory markers and the carotid plaque in patients with ischemic cerebrovascular disease. Chinese J Gerontol 2011; 31: 4773-4775.

11. Testelmans D, Tamisier R, Barone-Rochette G, Baguet JP, Roux-Lombard P, Pepin JL, Levy P. Profile of circulating cytokines: impact of OSA, obesity and acute cardiovascular events. Cytokine 2013; 62: 210-216.
12. Liu L, Wang X, Zhao L. Analysis of carotid atherosclerotic plaque and risk factors of the eldly patients with cerebral infarction. J Mod Clin Med 2011; 37: 105-106.

13. Yang Y, Yang B, Huang X. Study on relation between blood-lipid and carotid plaques formation in Senile. Chinese J Integr Med Cardio/Cerebrovasc Dis 2011; 9: 1432-1434.

14. Wang J, Dong C, Jin X. Study on the correlation between carotid plaque formation and blood lipids. Med J Chinese People Arm Police Forces 2008; 19: 827-828.

15. Qiuming D, Wang C. Correlation between risk factors of carotid plaque formation and ischemic cerebrovascular disease. Chinese J Integr Trad West Med Intens Crit Care 2010; 17: 364-366.

16. Zhang X. Analysis on correlation between carotid plaque in patients with ischemic stroke and levels of homocysteine and blood lipids. Contemp Med 2014; 20: 53-54.

17. Lu X, Zhang H, Yan F. Analysis on correlation between level of serum homocysteine and cerebral infarction. Shaanxi Med J 2010; 39: 875-876.

18. Xue W. Correlation between blood homocysteine in patients with ischemic stroke and carotid plaques. Chinese Foreign Med Res 2013; 11: 131-132.

19. Yan M, Shengjun W, Fan X. Study on correlation between carotid ultrasound changes in patients with ischemic stroke and plasma homocysteine level. Shaanxi Med J 2011; 40: 1604-1606.

20. Weimar C, Diener HC, Alberts MJ, Steg PG, Bhatt DL, Wilson PW, REACH Registry Investigators. The Essen stroke risk score predicts recurrent cardiovascular events a validation within the Reduction of Atherothrombosis for Continued Health (REACH) Registry. Stroke 2009; 40: 350-354.

21. He Y. Correlation between plasma homocysteine and carotid atherosclerotic plaque in patients with ischemic cerebrovascular disease. Chinese J Pract Nerv Dis 2015; 18: 18-20.

22. Amin MR, Mohammad H, Ahmed KGU, Sarkar DN. CRP in patients with acute ischemic stroke in Bangladesh. J Med 2014; 15: 41-47.

23. Chung H, Jung YH, Kim KH, Kim JY, Min PK, Yoon YW, Choi EY. Diastolic velocity and future cardiovascular events in asymptomatic high risk patients. Korean Circ J 2016; 46: 72-78.

\section{*Correspondence to}

Weibo Zheng

Department of Clinical Laboratory

Shaoxing Municipal Hospital

PR China 